\title{
Carcinoma de células en anillo de sello de glándula mamaria en canino
}

\author{
Seal ring cells carcinoma in mammary gland in caninne
}

López RR, Bazan Y. Carcinoma de células en anillo de sello de glándula mamaria en canino. Rev Colombiana Cienc Anim. Recia. 2020; 12(1):e734. DOI: https://doi.org/10.24188/recia.v12. n1.2020.734

Universidad de Sucre, Colombia

Los autores permiten a RECIA reimprimir el material publicado en él. En caso de que un autor quiera traducir o usar una publicación parcial o completa de nuestro Diario, el autor debe obtener un permiso por escrito del editor de la revista.

Copyright (C) 2020. El (los) autor (es), Revista Colombiana de Ciencia Animal - RECIA. 2020. Este es un artículo de acceso abierto distribuido bajo los términos de Creative Commons Attribution 4.0 (https://creativecommons.org/licenses/by-nc-sa/4.0/), El uso, distribución o reproducción está permitido, siempre que se acrediten al autor original y al propietario del copyright y que se cite la publicación original en esta revista, de acuerdo con la práctica académica aceptada. No se permite el uso, distribución o reproducción que no cumpla con estos términos. 


\title{
Carcinoma de células en anillo de sello de glándula mamaria en canino
}

\author{
Seal ring cells carcinoma in mammary gland in caninne
}

\section{Raúl López Ramos, M.Sc.}

Universidad Autónoma Gabriel René Moreno, UAGRM, Facultad de Ciencias Veterinarias, Laboratorio de Patología Veterinaria, Santa Cruz, Bolivia.

raullr20@gmail.com

(D) https://orcid.org/0000-0001-7364-4163

\section{Yaqueline Bazan, MVZ}

Universidad Autónoma Gabriel René Moreno, UAGRM, Facultad de Ciencias Veterinarias, Hospital de Escuela Veterinaria (HEV), Santa Cruz, Bolivia. yaquevet@hotmail.es (iD https://orcid.org/0000-0001-5593-3551
DOI: https://doi.org/10.24188/recia.v12.n1.2020.734

\section{RESUMEN}

Los tumores de glándula mamaria son el tipo de neoplasia más frecuente en hembras de la especie canina. Pueden presentarse como masas únicas o múltiples afectando varias glándulas mamarias, se clasifican según el tejido de origen y sus características histológicas en carcinomas, sarcomas y tumores mixtos, con frecuencia suelen presentarse varios tipos histológicos de forma simultanea afectando diferentes glándulas mamarias en el animal. Existen varios tipos de carcinomas según la clasificación de la OMS 1999 y la de Glodschmidt 2011, con valor pronóstico para el paciente. Se presenta un caso de carcinoma de células en anillo de sello en glándula mamaria de una perra de raza rottweiler, de 8 años de edad con presentación múltiple en mamas abdominales e inguinal derecha, siendo un tipo de carcinoma poco frecuente en glándula mamaria. La paciente presentó una sobrevida de 6 meses post-cirugía, con recidiva notoria a los 4 meses y una evolución de 14 meses desde la fecha de aparición del tumor.

Palabras Claves: Carcinoma, glándula mamaria, carcinoma de células en anillo de sello

\begin{abstract}
Mammary gland tumors are the most common type of neoplasm in females of the canine species. They can occur as single or multiple masses affecting several mammary glands, they are classified according to the tissue of origin and their histological characteristics in carcinomas, sarcomas and mixed tumors, often several histological types are presented simultaneously affecting different mamary glands in the animal. There are several types of carcinomas according to the WHO 1999 and that of Goldschmidt 2011 classification, with prognostic value for the patient. A case of seal ring cell carcinoma in the mammary gland of an 8-year-old rottweiler dog with multiple presentation in abdominal and right inguinal mammary gland. The patient presented a 6-month post-surgery survival and a 14-month evolution from the date the tumor appeared.
\end{abstract}

Keywords: Carcinoma, mammary gland, seal ring cell carcinoma 


\section{INTRODUCCIÓN}

Los tumores de glándula mamaria son el tipo de neoplasia más frecuente en hembras de la especie canina, y el segundo tomando en cuenta ambos sexos, afectan en un 97-100\% a hembras y 0-3\% en machos. Las hembras no castradas son más predisponente a padecerlos lo que indica la importancia del factor hormonal en la carcinogénesis. Se presentan a partir de los 4 años en adelante con una mayor acentuación entre los 8 y 10 años, afectan con mayor frecuencia a las mamas caudales, aproximadamente el $50 \%$ son de comportamiento benigno y el $50 \%$ de comportamiento maligno (1,2) aunque hay estudios que reportan una mayor incidencia de neoplasias malignas sobre las benignas $(3,4)$.

Los tumores mamarios pueden presentarse como masas únicas o múltiples y cuando existen varios tumores simultáneos estos pueden ser de diferente tipo histológico (5). Existen varios tipos de tumores mamarios agrupados según sus características histológicas, pueden ser de origen epitelial, mesenquimal o mixta. De acuerdo a la clasificación de la OMS 1999 hay 9 tipos de carcinomas ordenados de menor a mayor malignidad, "Carcinoma in situ, Carcinoma complejo, Carcinoma tubulopapilar simple, Carcinoma sólido, Carcinoma anaplásico, Carcinoma de células fusiformes, Carcinoma de células escamosas, Carcinoma mucinoso y Carcinoma rico en lípidos", lo que resulta, junto con el grado histológico de malignidad, en un factor pronóstico importante (6).

Goldschmidt et al (7) propone una nueva clasificación para tumores de glándula mamaria en la que se incluye nuevos tipos de carcinomas como el carcinoma mixto, carcinoma papilar intraductal, carcinoma cribiforme, comedocarcinoma y el carcinoma micropapilar. En un estudio reciente en la ciudad de Quito, basados en esta clasificación, Martínez et al (8) determinó que los tumores mamarios más frecuentes fueron el carcinoma tubulopapilar simple con $21.4 \%$ (n=28), carcinoma mixto con $17.9 \%$ y carcinoma complejo con $14.3 \%$.

Los tumores mamarios en perras se asemejan bastante a los que se presentan en la mujer, en ambas especies la incidencia se incrementa con la edad, aunque se registra una mayor incidencia de tumores mamario en perras con relación a la mujer, los tipos histológicos más comunes son de origen epitelial y tienen un patrón de metástasis a linfonódulos regionales y pulmón (9).

En la mujer, el carcinoma de células en anillo de sello primario de glándula mamaria, es un tumor poco frecuente, representa aproximadamente $0.7 \%$ del total de los carcinomas de mama, es considerado un tumor de comportamiento muy agresivo con un patrón inusual de metástasis hacia el tracto gastrointestinal lo que dificulta el diagnóstico diferencial con el carcinoma de células en anillo de sello de origen gastrointestinal $(10,11)$.

A continuación se describe un caso de carcinoma de células en anillo de sello en glándula mamaria, de una paciente canina de raza rottweiler cuya sobrevida luego de la cirugía fue de 6 meses.

\section{CASO CLÍNICO}

Historia Clínica. Se trata de un canino hembra de raza rottweiler con 8 años de edad, no castrada que se presenta a consulta en el HEV el 25 de julio 2018 por presentar tumores mamarios con 8 meses de evolución.

A la anamnesis se evidenció 3 tumores mamarios ubicados en las mamas abdominales e inguinales del lado derecho (Figura 1). El estado general sin alteraciones relevantes, sin decaimiento, apetito normal, dieta a base de balanceado y comida casera, con $44 \mathrm{~kg}$ de peso.

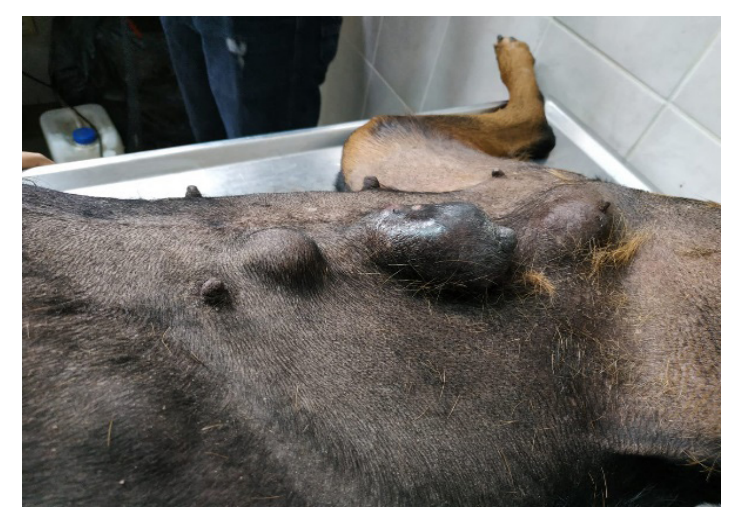

Figura 1. Tumores en las mamas adominales e inguinales del lado derecho 
Análisis complementarios y Cirugía. El hemograma y las pruebas de coagulación se encontraron dentro de los parámetros de referencia.

La ecografía y radiografía no evidenció indicios de metástasis en otros órganos.

Teniendo todos los análisis en valores normales, se procedió a realizar la cirugía más ovario histerectomía el 30 de Julio de 2018, el tipo de cirugía realizada fue mastectomía regional unilateral, extirpando las 3 últimas mamas con margen lateral de 2,5 $\mathrm{cm}$ y $1 \mathrm{~cm}$ de margen profundo.

Estudio Anatomopatológico. El examen macroscópico describe cadena mamaria derecha con 3 tumores de forma redondeada con un diámetro de $3 \mathrm{~cm}, 4.5 \mathrm{~cm}$ y $2.6 \mathrm{~cm}$ ubicados en la 1ra y 2da glándula abdominal e inguinal respectivamente. El nódulo inguinal se acompaña de 3 nódulos de $1 \mathrm{~cm}$ alrededor y 1 linfonódulo agrandado. Todos los tumores compuestos por un parénquima homogéneo color blanco grisáceo, firme y en el más grande con material purulento central (Figura 2).

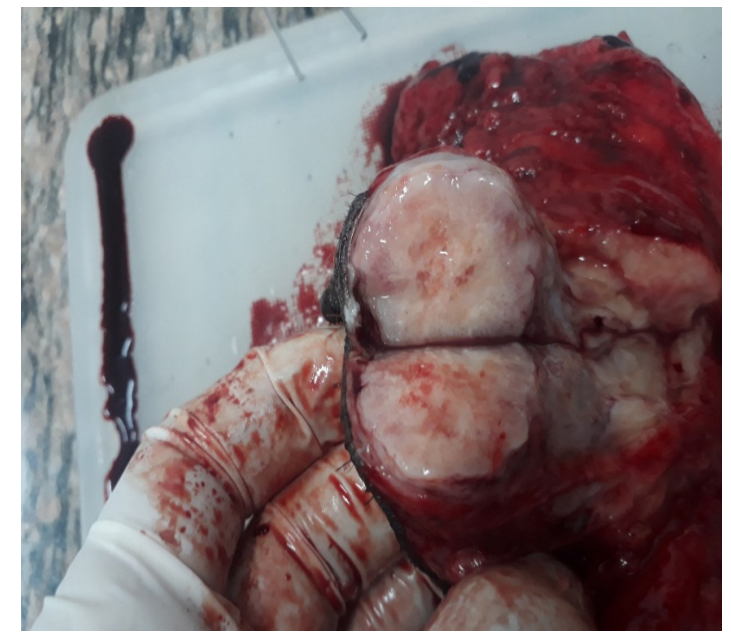

Figura 2. Tumor con corte transversal, muestra un parénquima blanco grisáceo, firme de bordes regulares.

El examen histopatológico evidenció una población sólida de células epiteliales dispuestas en pequeños nidos, sábanas e hileras separadas de abundante tejido fibroso o desmoplasia, muchas de las células (80\%) con núcleo excéntrico en forma de medialuna y citoplasma claro bien delimitado PAS+ mostrando presencia de mucina intracitoplasmática. Se evidenció las mismas células en linfonódulo regional inguinal (Figura 3, 4 y 5).

El diagnóstico fue carcinoma de células en anillo de sello con macrometastasis a linfonódulo inguinal.

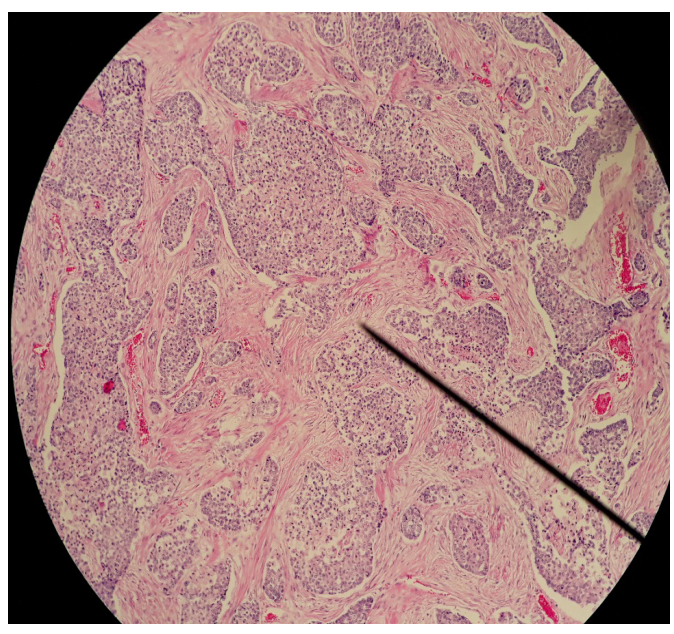

Figura 3. Carcinoma de células en anillo de sello 10x, muestra disposición sólida con abundantes desmoplasia.

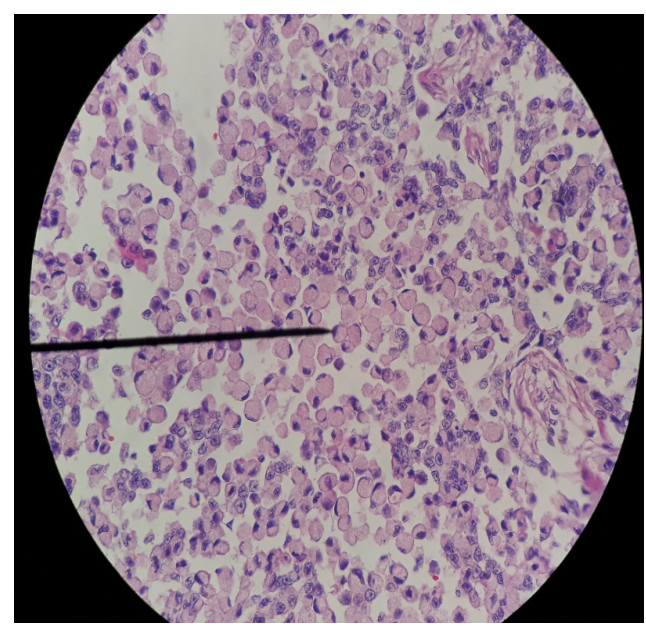

Figura 4. Carcinoma de células en anillo de sello 40x, células neoplásicas con núcleos excéntricos en forma de media luna y citoplasma eosinofílico bien delimitado. 
Seguimiento. Al 16 de agosto el animal vuelve a consulta para retirada de puntos, la herida se encuentra en estado de cicatrización normal. Se aprovechó de realizar otra radiografía, ecografía y hemograma de control.

La radiografía y ecografía no mostró evidencias de metástasis, las químicas sanguíneas para funcionalidad renal y hepática dentro de parámetros normales. El hemograma muestra una leucocitosis neutrofílica marcada de 25155 (V.R. 3000 $13500 \mathrm{Leu} / \mu \mathrm{l}$ ) y trombocitosis marcada. El propietario no accedió a realizar ningún protocolo quimioterápico adyuvante.

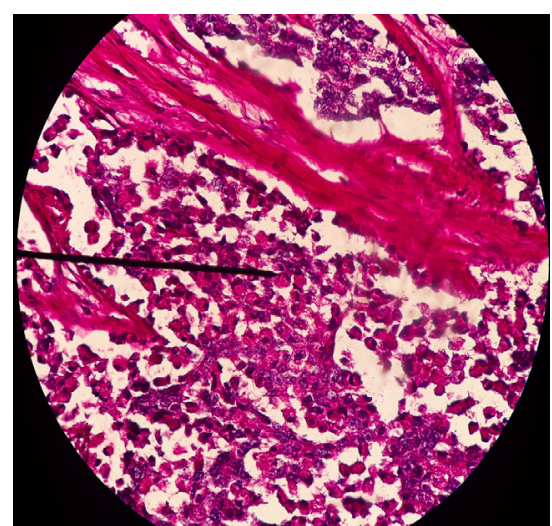

Figura 5. Carcinoma de células en anillo de sello 40x, tinción PAS+ intracitoplasmatica.

El 30 de agosto, un mes después de la cirugía, el paciente regresó para corrección de hernia abdominal y problemas de cicatrización de la herida producto de la cirugía, se retiró fragmentos de piel lesionada para la corrección de la cicatriz, este tejido se envió al laboratorio de patología FCV-UAGRM, se encontró un foco de 1 mm de diámetro con células neoplásicas recidivantes pero con un $20 \%$ de células en anillo de sello (Figura 6), por lo que se advirtió al propietario de la alta posibilidad de recidiva local y metástasis a distancia.

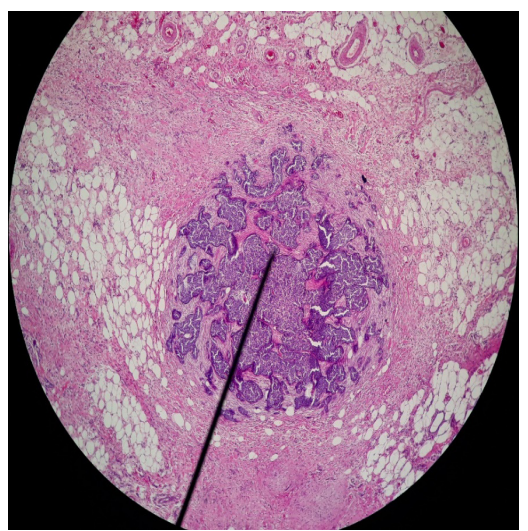

Figura 6. Carcinoma de células en anillo de sello 4x, recidiva 30 días post cirugía

A los 6 meses después de la cirugía el propietario comunica el fallecimiento del animal, mismo que había presentado recidiva local de las neoplasias dos meses antes (Figura 7).

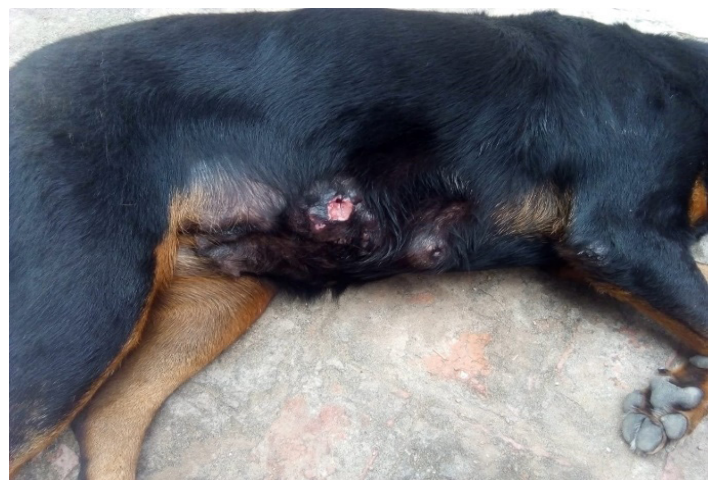

Figura 7. Carcinoma de células en anillo de sello, recidiva a 6 meses post cirugía. 
Finalmente no se tuvo acceso al animal para realizar la necropsia, el encontrarse a $65 \mathrm{~km}$ de la ciudad de Santa Cruz de la Sierra fue difícil el traslado del animal hasta el laboratorio de patología.

\section{DISCUSIÓN}

El carcinoma de células en anillo de sello primario de glándula mamaria en la mujer se considera un subtipo del carcinoma lobulillar infiltrante con alto grado de malignidad, aunque algunos tipos de carcinoma ductal suelen presentar áreas de diferenciación a células en anillo de sello, que indicaran un comportamiento más agresivo (12). En la clasificación de tumores mamario de caninos no se encuentra este subtipo y no se ha encontrado reportes de su presentación en perras de nuestra región, presumimos que por sus características tal vez sea clasificado dentro de los carcinomas sólidos de acuerdo a la clasificación de tumores mamarios para perras (7). El carcinoma de células en anillo de sello ha sido reportado entre los adenocarcinomas gástricos en caninos con vómito persistente, anorexia y pérdida de condición corporal con metástasis a linfonódulos perigástricos, bazo y omentos. $(13,14)$.

El diagnóstico confirmativo de carcinoma de células en anillo de sello primario de glándula mamaria se lo realiza mediante inmunohistoquímica, CK7 positiva para tumores primarios de mama y CK20 positiva para tumores de origen gástrico (11). Para este caso, no contamos con dichos marcadores para realizar la diferenciación, aunque asumimos que se trata de un carcinoma de células en anillo de sello primario de glándula mamaria por la ausencia de afectación en órganos de cavidad abdominal mediante ecografía y la falta de signos gastrointestinales que suelen observarse en tumores gástricos.

Los lugares más frecuentes de metástasis descritos para este tipo de carcinoma en mujeres son el sistema gastrointestinal y en ocasiones genitourinario (16). Por la dificultad para transportar el cadáver hasta el laboratorio, no se pudo realizar la necropsia del animal para evidenciar los sitios de metástasis a otros órganos.

Para el comportamiento post quirúrgico se confirmó una recidiva microscópica a los 30 días y una recidiva notoria a los 4 meses después de la cirugía, con una sobrevida de 6 meses luego de la extirpación y 14 meses desde la fecha de aparición, mostrando un comportamiento agresivo para este caso en particular, sin embargo se tendrá que realizar seguimientos a más casos similares para determinar mejor el comportamiento del tumor aunque es de escasa presentación, siendo el primer caso diagnosticado en el Laboratorio de Patología FCV - UAGRM.

Aunque el dueño no accedió realizar ningún tratamiento quimioterápico coadyuvante a la cirugía, los protocolos para este tipo de tumor en medicina humana están a base de adriamicina y ciclofosmamida, también se podría utilizar como adyuvante a la cirugía protocolos quimioterápicos generales para tumores mamarios en caninos a base de Doxorubicina en dosis de $30 \mathrm{mg} / \mathrm{m} 2$ IV seguida de Ciclofosfamida en dosis de 50mg/m2 PO $(11,5,15)$.

\section{CONCLUSIÓN}

Es importante realizar el estudio histopatológico de todos los tumores mamarios con el fin de determinar el tipo histológico del tumor y el grado histológico de malignidad, que junto al estudio de los márgenes quirúrgicos, son de mucho valor pronóstico para los tumores mamarios en medicina veterinaria.

La cirugía suele ser la primera opción de tratamiento siempre que sea posible la resección completa con márgenes limpios y sin evidencia de metástasis, pero en tumores de alto grado, esta tiene que ser acompañada de un protocolo quimioterápico a fin de conseguir una mejor calidad de vida y un mayor tiempo libre de enfermedad.

Es importante implementar pruebas de inmunohistoquímica para apoyar el diagnóstico de neoplasias en patología oncológica veterinaria, éstas permitirán el diagnostico confirmativo de muchos tumores y también aportarán datos indicativos de pronóstico del paciente.

\section{Conflicto de intereses}

Certificamos que no existe conflicto de intereses con ninguna organización financiera con respecto al material discutido en el manuscrito. 


\section{REFERENCIAS}

1. Sánchez CF, Guarín PC. Tumores de glándula mamaria en caninos. CONEXAGRO JDC. 2014; 4(2):60-78. https:// www.jdc.edu.co/revistas/index.php/conexagro/article/view/209/231

2. Cartajena Albertus JC. Oncología Veterinaria. 1ra ed. Navarra, España; Servet; 2011.

3. Chau VG, Chavera CA, Perales CR, Gavidia CC. Frecuencia de neoplasias en glándula mamaria de caninos: estudio retrospectivo en el periodo 1992-2006 en la ciudad de Lima, Perú. Rev Inv Vet Perú. 2013; 24(1):72-77. https://doi. org/10.15381/rivep.v24i1.1663

4. Torres VG, Botero EL. Estudio Histopatológico Retrospectivo de Neoplasias de Glándula Mamaria en Caninos (19752000). Orinoquia. 2008; 12(1):80-88. https://doi.org/10.22579/20112629.75

5. Hermo GA, Garcia MG, Torres PA, Gobello MC. Tumores de mama en la perra. Ciencias Veterinarias. 2005; 7(1):1-25. https://cerac.unlpam.edu.ar/index.php/veterinaria/article/view/1927/1880

6. Baba AI, Catoi C. Comparative Oncology. Bucharest: The Publishing House of the Romanian Academy; 2007. Chapter 11, Mammary Gland tumors. La editorial de la Academia rumana: Bucarest, Rumania; 2207. Disponible en: https:// www.ncbi.nlm.nih.gov/books/NBK9542/

7. Goldschmidt M, Peña L, Rasotto R, Zappulli V. Classification and Grading of Canine Mammary Tumors. Veterinary Pathology. 2011; 48(1):117-131. https://journals.sagepub.com/doi/full/10.1177/0300985810393258

8. Martínez-Trejo C, Asqui Aguayo S. Clasificación histopatologica de tumores mamarios en caninos extraídos quirurgicamente, en 6 clínicas veterinarias de la ciudad de Quito [Trabajo de Grado]. Quito: UCE; 2017. http://www. dspace.uce.edu.ec/handle/25000/13739

9. Caicedo JA, Iregui CA, Cabarcas ME, Acosta BJ. Estudio comparativo de la frecuencia de tumores mamarios según sexo, edad y tipo histológico en caninos y humanos en los laboratorios de patología anatómica de la Universidad Nacional de Colombia sede Bogotá. Revista Colombiana de Ciencia Animal. 2012; 5(1):37-46. http://revistas.ut.edu. co/index.php/ciencianimal/article/view/124/123

10. Muños V, Prince J, Mora E, Mahmoud B, Guerrero D, Moro J. Carcinoma de células en anillo de sello primario de glándula mamaria. Rev Venez Oncol. 2018; 30(1):10-15. http://www.oncologia.org.ve/site/upload/revista/ pdf/03. $m$ v (10-15).pdf

11. Jiménez M, Hierro M, Sánchez Ch, Espadas P. Carcinoma de células en anillo de sello primario de mama. Diagnóstico diferencial inmunohistoquímico. Revista Senología y Patol Mam. 2003; 16(2):70-74. https://www.elsevier.es/esrevista-revista-senologia-patologia-mamaria-131-articulo-carcinoma-celulas-anillo-sello-primario-13047778

12. Rubiales AS, Álvarez GT, Rey P, Del Valle ML, Uña E, Gómez L. Carcinoma de células en anillo de sello extramamario tras cáncer de mama: ¿Cuándo se trata realmente de un segundo primario?. Oncología. 2005; 28(9):448-452. http:// scielo.isciii.es/scielo.php?script=sci arttext\&pid=S0378-48352005000900005

13. Hernández C, Naranjo R, Acevedo M, Aranzazu D. Carcinoma gástrico de células en anillo de sello en un perro: Reporte de un caso. Rev Col Cienc Pec. 2004; 17(2):175-181. https://aprendeenlinea.udea.edu.co/revistas/index. $\mathrm{php} / \mathrm{rccp} / \mathrm{article} / \mathrm{view} / 323938 / 20781118$

14. Ortiz AJ. Adenocarcinoma gástrico con metastasis a hígado y pulmón en un canino. Journal of Agriculture and Animal Sciences. 2013; 2(2):68-77. http://repository.lasallista.edu.co:8080/ojs/index.php/jals/article/view/610/349

15. Issotta C, Weinborn R, Agurto M, Lara J. Caso Clínico: Tratamiento de carcinoma tubulopapilar de glándula mamaria a través de masectomía radical bilateral completa y quimioterapia en un paciente canino. Hospitales Veterinarios. 2015; 7(2):52-56. http://www.rhv.cl/index.php?option=com docman\&task=doc download\&gid=108\&Itemid=.

16. Mucientes F, Villalobos P, Klaassen V, Murcientes P, Heredia F, Villanueva J, Landeros J. Metástasis de carcinoma de células en anillo de sello al cuello uterino. Rev Chil Obstet Ginecol. 2013; 78(3):229-235. https://scielo.conicyt.cl/ pdf/rchog/v78n3/art10.pdf 